\title{
Notes sur d'éventuelles influences du « Journal de l'année de la peste » de Daniel Defoe sur «vagues souvenirs de l'année de la peste » De Jean-Luc Lagarce
}

\author{
Riham Mohammed Nabile Ibrahime Hifnawy* \\ r.hifnawy@ mu.edu.eg
}

\section{Résumé}

Jean-Luc Lagarce (1957-1995), l'un des dramaturges contemporains les plus joués en France. Il était connu de son vivant comme un metteur en scène mais un auteur méconnu. Il vivait par et pour le théâtre. Il écrivait vingt-huit pièces de théâtre. Sa pièce Vagues souvenir de l'année de la peste (1982), est une pièce inspirée de Journal de l'année de la peste de Daniel Defoe, un grand romancier anglais. Journal de l'année de la peste a été publié le 17 mars 1722 suite à la peste qui ravageait Marseille en 1720 et qui frappait Londres en 1664-1665. De fortes ressemblances entre le roman de Defoe et «Vagues souvenirs de l'année de la peste» méritent une attention particulière pour plusieurs raisons. Il faut prendre en considération également que les deux ouvrages sont fort éloignés, l'un de l'autre dans la tempe et l'espace, et peut-être dans les événements.

Cette présente étude tentera de passer en revue les fortes ressemblances entre les deux ouvrages. Puis elle étudiera comment Lagarce réussit à transformer un ouvrage classé sous la rubrique de l'art romanesque en une pièce de théâtre. Enfin elle essayera de montrer les particularités de

* Maître de conférences, Département de français ,Faculté de Lettres, Université de Minia

(Notes sur d'éventuelles influences du...)Dr. Rihame Hifnawy 
chacun des deux ouvrages où nous focalisons le regard sur les ressemblances et les dissemblances.

Mots clés: Lagarce , Defoe, peste, l’épidémie , réécriture

\section{Introduction}

On a beaucoup écrit sur le théâtre de Jean-Luc Lagarce (19571995), l'un des dramaturges contemporains les plus joués en France $^{1}$. Il était connu de son vivant comme un metteur en scène mais un auteur méconnu ${ }^{2}$, c'est probablement à cause du langage théâtral de son cuvre qui est en décalage, trop novateur ${ }^{3}$.

Des chercheurs assidus en France ainsi qu'à l'étranger ont tenté il y a plusieurs années de s'attaquer aux différents thèmes qui traversent l'œuvre dramatique de cet écrivain. Ils se sont arrêtés devant l'image de la solitude, de l'inquiétude, l'individualisme et des relations familiales.

À lire soigneusement les vingt-cinq pièces de Jean-Luc Lagarce, le lecteur attentif découvre aisément que cet écrivain (mort très jeune à l'âge de 38 ans) s'est remarquablement préoccupé du thème de la maladie et de la disparition. Nombreuses sont, en fait, les pièces qui en témoignent. Informé de sa séropositivité à la suite d'examens médicaux en 1988, JeanLuc Lagarce a passé les années suivantes à raffiner et à approfondir un thème cher à lui depuis sa petite jeunesse : la maladie.

(Notes sur d'éventuelles influences du...)Dr. Rihame Hifnawy 
Notons qu'un certain nombre des pièces de Lagarce sont inspirées des œuvres d'autres auteurs. C'est le cas de notre corpus, Vagues souvenir de l'année de la peste (1982), pièce fortement inspirée du grand maître du roman anglais Daniel Defoe $^{4}$, Journal of the Plague Year (1722) (Journal de l'année de la peste) reflète mieux que les autres pièces cette hallucination de la maladie et de la disparition dans l'œuvre de Jean-Luc Lagarce. Faute d'espace, nous limitons notre corpus à cette pièce, traduite en plusieurs langues, et qui montre, nous semble-t-il, comment ce dramaturge contemporain est classé parmi les écrivains de la « clairvoyance ».

Loin de le classer parmi les écrivains de l'absurde de l'époque qui sont presque unanimes à réfléchir sur le destin de l'homme contraint de naître pour mourir ${ }^{5}$, Lagarce a pu créer, et grâce à ses lectures et à sa vie remplie de tristesse, d'accentuer ce thème de la maladie et de la disparition.

Une lecture aussi profonde que possible nous a révélé que Lagarce s'est nourri largement de «A Journal of the Plague year » de Daniel Defoe. De fortes ressemblances entre le roman de Defoe et «Vagues souvenirs de l'année de la peste » méritent une attention particulière pour plusieurs raisons : tout d'abord par le fait que l'ouvrage de Defoe est classé sous la rubrique des créations romanesques et que celui de Lagarce est une pièce de théâtre. Il faut prendre en considération également que les deux 
ouvrages sont fort éloignés, l'un de l'autre dans la tempe et l'espace, et peut-être dans les événements. Enfin, il est utile de ne pas oublier que «Journal de l'année de la peste» de Defoe a été publié le 17 mars 1722 suite à la peste qui ravageait Marseille en 1720 et qui frappait Londres en 1664-1665, et que «Vagues souvenirs de l'année de la peste » a été publié quelques mois après la parution d'une célèbre traduction en français de «Journal of the plague year» de Defoe par Francis Ledoux en 1982.

En Somme, face à ces fortes ressemblances, plusieurs interrogations s'imposent : comment Lagarce a puisé dans «Journal de l'année de la peste » de Defoe pour créer son propre ouvrage «Vagues souvenirs de l'année de la peste»? Comment a-t-il réussi à transformer un ouvrage classé sous la rubrique du roman en pièce du théâtre ? a-t-il réussit à imiter librement l'auteur anglais tout en écrivant sa pièce de théâtre ? A-t-il adapté le roman de Defoe ou s'en est-il inspiré longuement ? Quelles sont les ressemblances et les dissemblances entre les deux ouvrages ?

A partir de ces questions surgit le sujet de la présente étude où, dans un premier temps, nous tenterons de passer en revue les fortes ressemblances entre les deux ouvrages. Dans un deuxième temps, nous étudierons comment Lagarce réussit à transformer un ouvrage classé sous la rubrique de l'art romanesque en une pièce de théâtre. Dans un troisième temps, nous essayerons de montrer

(Notes sur d'éventuelles influences du...)Dr. Rihame Hifnawy 
les particularités de chacun des deux ouvrages où nous focalisons le regard sur les ressemblances et les dissemblances.

\section{Le récit de l'épidémie}

De fait, Daniel Defoe avait cinq ans lors de la terrible épidémie de la peste de Londres. Son œuvre «A journal of the Plague year» a été décrite par un témoin qui a vécu durant l'épidémie à Londres et qui a refusé de quitter sa ville. C'est pour cette raison-là qu'on peut dire que cet ouvrage est basé sur les souvenirs d'enfance de Daniel Defoe, les dires des témoins de l'époque et les préjugés populaires. Mais la peste de Marseille de l'été 1720 a nourri Defoe pour écrire son roman qui a décrit l'épidémie du $18^{\mathrm{e}}$ siècle.

«Vagues souvenirs de l'année de la peste» de Lagarce est une réécriture du «A journal of the Plague year» de Defoe où Lagarce entreprendra de réécrire les textes d'autres écrivains ${ }^{6}$. Lagrace, lui-même a noté dans journal 1977-1990 que cette pièce est «relecture du Journal de l'année de la peste de Defoe $»^{7}$. En effet, dans le théâtre de Lagarce la réécriture apparaît comme le fil conducteur de l'écriture. On peut distinguer différents types de réécriture. Erreur de Construction, la première pièce de Jean-Luc Lagarce, écrite en 1977, est un moyen de rendre hommage à Eugene Ionesco et au théâtre de l'absurde. C'est une réécriture de La Cantatrice Chauve. Et la deuxième pièce de son répertoire, Carthage, Encore est inspirée de Samuel 


\section{Beckett et Voyage de Madame Knipper vers la Prusse Orientale} s'inspire du voyage d'Olga Knipper, épouse d'Anton Tchékhov qui partit chercher le corps de son mari. Quant à Vagues souvenirs de l'année de la peste présente des personnages reprenant les noms de personnages de l'œuvre de Daniel Defoe qui ont réellement existés.

Il est intéressant de noter que Journal of the plague year n'est pas la seule source d'inspiration de Jean-Luc Lagarce. Il faudra penser également à Albert Camus notamment à sa pièce L'état de Siège (1948). Dans cette pièce, Camus a inventé un mythe moderne pour développer une allégorie multiple, teintée de fantastique, sur les régimes corrompus, autoritaires, faschistes. L'action se déroule en Espagne, alors que dans la réalité en 1948, ce pays subit depuis plusieurs années la dictature franquiste. Alors, Son œuvre revêt une signification métaphysique, elle met l'accent sur tout ce qui est injuste dans le monde. Il faut signaler que L'état de Siège, est une pièce en trois parties, représentée pour la première fois en 1948.

Journal of the plague year de Daniel Defoe constitue une œuvre complète sur la peste. Il relate les diverses évocations de la maladie en commençant par la déclaration que la peste était revenue en Hollande en septembre 1664. Dans ce roman, Le narrateur $H . F^{8}$ est un sellier célibataire. Il a vécu à Londres pendant la peste qui ravageait le pays en 1665. Il hésitait entre deux décisions importantes "l'une, c'était mener (ses) affaires

(Notes sur d'éventuelles influences du...)Dr. Rihame Hifnawy 
qui étaient considérables et où était embarqué tout ce que (il) possédait en ce monde, et l'autre, c'était (se) sauver de cette horrible catastrophe qui, (il) le voyait bien menaçait tout la ville ${ }^{9}$. Cependant, il a finalement décidé de rester pour sauver tout son fonds et tout ce qu'il avait au monde. Le roman est un recueil de ses écrits et ses observations sur cette époque. Il a eu du mal à rester chez lui, Il parcourait la ville pendant les mois de la peste, parlant parfois avec d'autres Londoniens de leurs expériences. Et même dans les périodes où il vit cloîtré chez lui, H.F a passé ses journées à sa fenêtre à observer la Cité « pour voir comment la maladie se propage, puis décline, et comment les habitants réagissent à son avancée ${ }^{10}$.

En transformant une relation minutieuse de la réalité en une œuvre d'art, Daniel Defoe a décrit soigneusement la vie à Londres et les faubourgs en ce temps-là où «on voyait le chagrin, la tristesse sur toutes les figures, certains n'étaient pas encore abattus, mais tous paraissaient profondément anxieux, chacun se considérait, lui-même et sa famille, comme dans le plus grand danger $^{11}{ }$. Il a représenté la souffrance physique et psychologique des habitants : les malades qui ont subi la douleur de la peste ; les gens qui trouvaient que le seul moyen de survivre à la peste est de la fuir et d'autres qui se sont suicidés à cause de l'incapacité de savoir comment la peste s'est propagée ou comment ils peuvent s'en protéger. Ils se jetaient dans la fausse creusée pour enterrer 
les morts, «on les trouva morts en venant enterrer d'autres morts ${ }^{12} »$.

En effet, ce roman est une œuvre exceptionnelle. Il décrit les figures du malheur et de la douleur. L'auteur ne s'intéressait pas uniquement à rappeler les victimes de la peste et leur vie précaire mais il dépeignait des tableaux remarquables de charité, de générosité et d'humanité manifestées par les habitants de Londres pendant l'épidémie. Par exemple, une histoire de deux frères et leur père : un frère avait été soldat pendant les guerres civiles, ancien blessé ne pouvait pas faire un travail très pénible. Il avait été employé quelque temps dans une fabrique de biscuit de mer à Wapping. Il encourage son frère à quitter Londres pour trouver la sécurité et la santé dans un autre pays. Il est devenu le chef des trois hommes et le groupe de voyageurs auquel ils se sont joints, pace qu'il trouvait ingénieusement le moyen de traverser des villes inhospitalières. L'autre était Marin. Blessé aussi d'une jambe, il ne pouvait plus être embarqué. Pour gagner sa vie il travaillait comme ouvrier voilier à Wapping. Leur père était Charpentier, il emportait toujours avec lui sa boîte à outils partout. Cette boîte représentait toute sa richesse. Les trois hommes étaient restés dans la Cité très tard pour pouvoir fuir vers l'Est avec un groupe de pauvres voyageurs en provenance de Londres. Pour le narrateur, l'histoire de ces trois hommes paraît «un très bon exemple à suivre pour tout homme sans grands moyens, au cas où pareille Calamité publique reviendrait, et si

(Notes sur d'éventuelles influences du...)Dr. Rihame Hifnawy 
telle chose n'arrive pas, ce que Dieu veuille (nous) accorder dans sa Miséricorde infinie ${ }^{13}{ }$.

Dans son roman, Daniel Defoe a développé les réactions des Londoniens contre les effets de la peste : comment ils ont allégé la souffrance des autres, comment ils se sont tournés vers Dieu et ont confessé sincèrement leurs péchés. Ils n'ont pas abandonné leur foi en Dieu. Ils ont triomphé d'une terrible catastrophe par leur volonté, leur vertu et leur patience. De même le personnage nommé Heath, un médecin qui était un ami de H.F. à qui le narrateur rendait de fréquentes visites pendant la peste pour les préservatifs contre la contagion. C'était un bon Chrétien qui a fourni à H.F un grand secours dans ces temps terribles. Lorsque l'épidémie faisait rage dans le quartier, Il a encouragé HF à rester enfermé chez lui et de ne plus sortir pendant toute la durée de la peste. «Fermer les fenêtres, les volets et les rideaux, maintenir une épaisse fumée nourrie de poix et de résine, de poudre à canon et de soufre dans la chambre où on serait obligé de tenir fenêtre ou porte ouverte ${ }^{14}{ }$.

Mais contrairement à de nombreux écrivains qui ont décrit leur désapprobation face aux pauvres qui ne peuvent pas se contrôler durant cette période de grands troubles, Defoe refuse de suivre ce chemin: son narrateur fait preuve d'une sympathie et d'une compassion pour les pauvres de Londres. Il montre que beaucoup de leurs moyens de subsistance ont été affectés par la peste et qu'ils peuvent à peine nourrir leurs familles. Alors, 
«Journal of the plague year » est un récit en forme de reportage sur la peste de Londres (1665) que Daniel Defoe n'a pas vraiment vécu. Mais il a réussi à écrire un roman homodiégétique «désigné par des initiales (H.F) construit un système axiologique et aléthique mimétique de celui du témoignage ${ }^{15}{ }$.

\section{Du roman à la pièce}

De ce roman Jean-Luc Lagarce a puisé son inspiration pour écrire Vagues souvenirs de l'année de la peste, une pièce qui traite le même événement, mais cette fois un an après avoir fui la peste de Londres. Les personnages de la pièce sont au nombre de huit personnages rescapés de cette maladie contagieuse et mortelle. Ces survivants qui se sont arrêtés chaque soir pour camper ensemble par la force des choses. Ils forment un campement aux abords de la ville dans l'espoir de pouvoir enfin y retourner. Ils tentent de survivre dans une société humaine où chacun a son rôle. Mais c'est exactement le contraire dans le roman de Defoe, ils ne réussissent jamais à vivre ensemble dans la catastrophe. Parce que dans le premier monologue dans cette pièce, chaque personnage donne un avis différent sur la fuite de Londres, ce qui engendre les conflits entre eux. Ces personnages racontent des fragments de récits après que «la peste avait achevé son auvre ${ }^{16}$; ils sont réunis dans l'errance, ils reprennent l'évènement où Defoe s'est arrêté : "personne ne s'inquiétait encore ... plus qu'il n'est nécessaire en pareil cas ... de savoir si l'épidémie cessait le plus naturellement du monde, par la

(Notes sur d'éventuelles influences du...)Dr. Rihame Hifnawy 
lassitude de la mort... ou par manque de victime ... faute de combattant se livrant à elle... $\gg^{17}$.

Le plus souvent, les personnages du théâtre Lagarcien ne vivent pas leur drame mais ils racontent comment ils l'ont vécu. Ils recomposent leurs histoires. Ils décrivent et commentent les faits passés. C'est le cas aussi de Vagues souvenirs de l'année de la peste. Jean-Luc Lagarce donne différents types de personnages de milieux différents qui racontent leurs histoires à cette époquelà. Dans cette errance, les personnages parlent de leur vie passée. Ils décrivent leur départ et leurs espoirs de retourner vers la ville de Londres, sains de corps et d'esprit comme ils vivaient auparavant. Par exemple; Le couple aristocratique Monsieur et Madame Forster qui ne parlent que de leur départ et de leur inquiétude à laisser leurs possessions derrière eux. Ils trouvent qu'il n'est pas raisonnable d'attendre une telle fin. Ils élaborent le plan de départs, et mettent leurs affaires en un lieu sûr :

«Trouvons une traite paisible où nous serons certains de les retrouver quand nous reviendrons et mettons-nous en route ... car nous reviendrons, n'en doutons pas, c'est pour cette unique raison que nous partons $»^{18}$

À l'aide de ces personnages, Jean-Luc Lagarce présente une parodie du monde aristocratique conservateur. Ce couple raconte partout comment il a laissé avec regret et avec prudence 
tous ses biens matériels partant vers des territoires plus cléments pour éviter la peste et ses tristes conséquences :

Monsieur Forster: L'Angleterre, en elle-même, comme son nom l'indique et il n'est pas besoin de revenir làdessus, l'Angleterre était à l'abri de toute épidémie, quelle qu'elle soit. Tout le monde sait cela, et les habitants de l'Angleterre, par définition, le savaient plus encore que tous les autres... (...) Jamais une quelconque épidémie, quelle qu'elle soit et quelle qu'elle puisse être, jamais une quelconque épidémie ne pouvait, n'aurait pu les atteindre... ${ }^{19}$.

L'image de ces époux âgés peut être une critique de la mentalité bourgeoise-capitaliste. Ce sont les propriétaires qui ne pensent qu'à reprendre leurs affaires :

Madame Forster: un jour, il faut le savoir, nous reviendrons, et cela ne saurait tarder...soyons optimistes: nous sommes déjà revenus... Quand ferons à nouveau notre entrée dans la ville de Londres, nous reprendrons notre rang, les affaires qui étaient les 
nôtres avant cette histoire, cette sale histoire... Nous n'avons rien à nous reprocher, et nous n'avons de comptes à rendre à personne ${ }^{20}$.

En effet, l'attitude de Monsieur et Madame Forester face à la catastrophe ressemble dans une certaine mesure à celle du frère de H.F dans le roman de Defoe. Il semble que l'idée de ces personnages lui avait été fournie par ce personnage de Daniel Defoe. Pour sauver sa vie, le frère de H.F est parti avec sa femme et ses deux enfants, il a quitté sa grande maison à Londres. Il ne s'est jamais préoccupé de ses affaires à Londres. Il a abandonné un entrepôt de nombreuses marchandises, y compris des chapeaux qui, pendant la peste, ont été volés par plusieurs femmes.

Il est évident que tous les fuyants ont quitté leurs propriétés précieuses derrière eux. Lagarce donne une autre image de cette attitude à travers un autre personnage : c'est La Jeune Femme qui raconte l'histoire du début de la peste et informe les spectateurs du nom de ce quartier où le premier cas est signalé et quand la peste commençait à peine à sévir. Elle se concentre toujours sur le début de la peste. Cette fois, la jeune femme a quitté son compagnon à Londres, "lui, il (m’) a dit de partir la première ... partir la première et (me) mettre à l'abri ${ }^{21}{ }$ après la mort du troisième cas par crainte de l'épidémie. Dans la pièce, la jeune femme décrit un spectacle terrifiant après la mort du troisième cas et lorsque la mort devint simultanée, «ils tombaient 
partout, dans les coins de la ville de Londres ${ }^{22}{ }^{\prime}$. C'est la même image présentée par Daniel Defoe lorsque deux hommes meurent de la peste dans Long Acre ou vers le haut de Drury Lane, à l'ouest de Londres, la terreur s'est répandue dans Le Cité. Le gouvernement tint plusieurs conseils sur les manières d'empêcher la peste de ravager Londres. Cependant les gens observaient que les listes des morts dans toute la ville s'allongent considérablement: "c’était là un triste épisode, mais tout le spectacle était plein de terreur, la charrette portait seize ou dixsept cadavres enveloppés de draps ou de couvertures $\gg^{23}$

Il est clair que dans sa pièce, Lagarce recourt à un autre événement historique relatif à cette époque pour montrer le destin d'un groupe de personnages qui parle de sa vie passée. Ils espèrent pouvoir rentrer dans la capitale, pour reprendre leurs maisons et leurs vies. Mais ces personnages, attirés par la lueur de la ville de Londres, se rapprochent des portes de la ville sans savoir que cette lueur est un grand incendie ou ce qu'on appelle Le Grande Feu de Londres ${ }^{24}$.

Le plus souvent d'ailleurs, Lagarce désigne ses personnages par leur statut comme L'Homme, La Jeune-Femme et La Fillette. D'autre part, il a recouru à des noms des personnages qui peuvent se retrouver d'un texte à l'autre. Dans Vagues Souvenirs de l'année de la peste, Lagarce n'a pas fait appel aux personnages du roman, mais il a présenté des personnages qui portent les mêmes noms que ceux de Daniel 
Defoe : Robinson Kreutiznaer et Molly ou Robinson crusoé et Moll Flanders dans l'œuvre de Daniel Defoe. Signalons que Lagarce n'a pas oublié de mentionner le nom de Daniel Defoe au début de sa pièce alors qu'il identifie le personnage de l'Homme $^{25}$. Ce personnage, qui joue le rôle de narrateur avec un monologue prologue, nous fournit des indices spatio-temporels de cet événement :

À la fin de cette année-là... nous devions déjà être au beau milieu du mois d'octobre... ou même, au milieu du mois de novembre... l'automne... il pleuvait, la pluie et le vent ... à la fin de cette année-là qui ... comme chacun sait (...) nous pouvions entendre que dans la ville de Londres, la peste avait achevé son œuvre... ${ }^{26}$

Le personnage du narrateur dans la pièce est, en grande partie, inspiré par l'auteur anglais mais L'Homme, le narrateur dans Vagues souvenirs de l'année de la peste, raconte les faits passés avant la fuite de l'épidémie et durant cette année d'errance. Il décrit tous les événements qui se sont passés à chaque nouveau campement. Il annonce de nouveaux événements :

«C'est ici que tout s'est terminé ... que tout a pris fin .... La dernière nuit, après une longue année d'errance 
dont nous doutions parfois de voir la fin... $\gg^{27}$

Dans Journal de l'année de la peste, la narrateur H.F. transforme son expérience sensorielle en écriture, il raconte de nombreuses histoires des habitants de Londres qui ont été touchés de la peste. Les histoires des parents qui ont perdu leurs enfants et celles des plus riches qui s'empressaient de quitter la Cité de Londres avec leurs familles; de la Cour qui était partie de bonne heure en juin pour Oxford; des pauvres qui décidaient de rester dans la ville et les histoires des Astrologues qu'ils « interprétaient comme précurseurs de sécheresse, de famine et de peste ${ }^{28} \gg$.

Vagues Souvenirs de l'année de la peste est une pièce parabole, dans laquelle Lagarce fait intervenir également le personnage de Robinson Kreutznaer, incarné par un homme d'une cinquantaine d'années. En conservant l'orthographe germanique du nom de Crusoé, il rappelle les origines de Robinson dont le père est de Brème. Ce marin aventurier est un suspect auprès des autres personnages. Ils se méfient de lui, parce qu'il est peut-être hollandais comme le navire qui a apporté la peste. Robinson Kreutzner ou Robinson Crusoé ${ }^{29}$ est le nom du protagoniste de l'un des romans les plus importants et les plus célèbres de Defoe $^{30}$. Robinson Crusoé, publié en 1719 , raconte la survie d'un naufragé sur une île déserte. L'histoire de ce roman est inspirée de l'aventure d'Alexandre Selkirk, un marin écossais qui a débarqué sur l'île inhabitée. Le roman traite de la bourgeoisie marchande et 
de l'utilitarisme en Angleterre du dix-huitième siècle. Dans la pièce, Il fait lui-même référence à sa soif de voyager, de visiter le monde comme marin :

"Je suis arrivé dans le port de la ville à un mauvais moment (...). Je voulais visiter le monde, j'aurais bien aimé, mais ce n'était pas de circonstance, me répondait-on. Pas un navire ne quittait les quais : les autres pays ne souhaitaient pas vraiment toucher à nos marchandises $\gg .^{31}$

Ce personnage aux origines lointaines évoque aussi le thème de l'étranger et une xénophobie subtile qui apparaît dans la pièce. Son nom de famille effraie les autres personnages qui se renseignent sur ses origines. Évidemment, La peur est directement liée à la peur de l'épidémie de peste.

Robinson K. - Évidemment... souvent on me demande... on me tient à distance, on ne veut pas que je m'approche: je peux être malade et paraître en bonne santé... je peux vouloir donner la maladie à tout le campement... On dit cela: il y a des gens qui sont malades et qui ont encore la force de marcher... (...) ou 
bien encore, je peux être atteint de la peste et ne pas le savoir... On me demande... les gens me demandent souvent cela... on me demande: «Kreutzner, c'est votre véritable nom?...»

«Kreutzner, ce n'est pas votre véritable nom?... »

«Kreutzner, si vous voulez mon opinion... Kreutzner, ce n'est pas un nom anglais, pas que je sache... jamais entendu... un patronyme anglais... ${ }^{32}$

Dans Vagues Souvenirs de l'année de la peste, Molly s'inspire de l'héroïne d'un autre roman de Daniel Defoe Moll Flanders $^{33}$ (1722), fille d'une pauvre voleuse, née en prison de Newgate. À l'aide de ce personnage, Defoe a décrit les mœurs et les costumes de la société du $\mathrm{XVIII}^{\circ}$ siècle. Cette jeune fille est forcée de travailler pour gagner sa vie. Elle s'est mariée cinq fois pour acquérir la stabilité financière. Continuellement, elle ne vit que dans l'espoir de trouver un mari riche. Mais après avoir découvert qu'un de ses maris est son propre frère, Moll devint une voleuse et est envoyée à la prison de Newgate.

Jean-Luc Lagarce s'inspire de ce personnage qui souffrait depuis sa naissance à Newgate pour survivre et pour devenir riche 
et honnête. L'histoire de sa vie est le combat d'une femme qui a cédé à la tentation du vol, du sexe et de la trahison et qui essaie malgré tout de changer cette vie pour vivre honnêtement. Dans «vagues souvenirs de l'année de La peste » Lagarce fait référence à Moll Flanders. Molly raconte qu'elle est née à New Gate et qu'elle était là lorsque la peste a commencé. C'est un personnage rejeté par tous les autres. Comme tous les personnages de la pièce, elle a fui la peste de Londres un an plus tôt. Elle partait pour survivre, elle cherchait à joindre le campement aux bords de la ville dans l'espoir de pouvoir y retourner. Cependant, après une aventure pénible, marchant sous la pluie, elle faisait «ce grand retour vers la ville de Londres ... les pieds un peu crottés ${ }^{34}$. Elle a appris que le campement est au complet, et qu'il n'y a pas de place pour elle :

«Notre communauté est au complet et nous ne pouvons rendre la vie plus difficile à ces braves gens ... à cette fillette qui a besoin de repos et d'espace ... (...) vous-même, ce n'est pas le moindre de mes soucis, vousmême, vous passeriez une très mauvaise nuit... ${ }^{35}$

Il est important de mentionner que Lagarce a recours à Molly pour décrire le drame de la peste. Elle se souvient toujours des histoires et les inclut dans son propre discours :

(Notes sur d'éventuelles influences du...)Dr. Rihame Hifnawy 
«Quand ça a commencé. (...) quand on nous racontait que, dehors, ils tombaient comme de pauvres mouches de rien. (...) nous n'avons pas ri longtemps. Pour enlever les cadavres, pour les envelopper dans des sacs et les mettre en terre, c'est à nous qu'ils ont pensé » 36

Dans Journal de l'année de la peste de Defoe, le narrateur raconte la même souffrance à travers des histoires semblables : c'était là un triste épisode, tout le spectacle était plein de terreur, «la charrette portait seize ou dix-sept cadavres enveloppés de draps ou de couvertures, quelques-uns si mal recouverts qu'ils tombèrent nus parmi les autres $»^{37}$. En effet, Lagarce ne fait pas seulement intervenir les personnages de Defoe et leurs souvenirs mais aussi les histoires de la peste.

On ne peut pas ignorer que la pièce de Lagarce est chargée de nombreux thèmes à travers ce groupe de personnes qui sont ensemble dans la catastrophe, et qui sont incapables d'une réelle coexistence et coopération, ils représentent l'égoïsme et l'individualisme de l'humanité. Et nous trouvons dans la pièce de nombreux signes qui soulignent la possession d'objets, la protection de biens matériels. Ce qui reflète une mentalité bourgeoise-capitaliste, surtout quand elle présente la bourgeoisie comme classe dominante. Tous ces thèmes se rattachent aux

(Notes sur d'éventuelles influences du...)Dr. Rihame Hifnawy 
thèmes abordés par l'écrivain anglais Daniel Defoe, évoqué comme personnage de la pièce de Lagarce.

Il faut noter que cette pièce est une pièce (palimpseste). Dans laquelle, On peut trouver la trace de l'œuvre de Daniel Defoe. Mais Lagarce conserve encore son style d'écriture. Dans Vagues Souvenirs de l'année de la peste, il est impossible d'enregistrer une véritable intrigue. Il ne se passe rien. Il n’y a plus de construction collective d'une histoire. Les répliques des personnages sont formées de fragments de récits plutôt que de dialogues. Chaque personnage donne des monologues qui représentent ses pensées face à un événement catastrophique comme l'épidémie de la peste. Ces monologues se rapprochent de la conception d'un «monologue intérieur», qui «ouvrait une crise du récit. Le hasard des associations d'idées se substituait à l'ordonnance de la narration. Avenir, présent et passé se confondaient ; les pensées étaient mêlées aux sensations provoquées par le monde extérieur $»^{38}$. Chaque personnage possède sa propre perception, il se nourrit d'elle et la promeut dans un monologue infini.

On peut dire également que tout est dans la langue et la parole. Les répliques des personnages tentent autant que possible de se rapprocher du langage oral. Leur parole imite la parole orale dans toutes caractéristiques: la répétition, l'hésitation et les pauses continuelles à l'intérieur du discours qui sont signalées par trois points :

(Notes sur d'éventuelles influences du...)Dr. Rihame Hifnawy 
MONSIEUR FORESTER: En nous enfuyant... parce que tout de même... il faut le reconnaître... (...)... En nous enfuyant... MADAME FORESTER, surtout, $\quad \mathrm{y}$ tenait $\quad \ldots \quad$ c'est compréhensible: loin de moi l'idée, aujourd'hui de le lui reprocher... La pauvre femme... MADAME FORESTER... la pauvre femme n'était pas rassurée, mais la situation, qui en douterait ? (...) ...

Donc, nous partons.

Et nous enfuyant... parce que, après tout... qui aurait fait autrement ?... C'est aussi cela la question... posons la question; (...) Madame Forster et moi, nous savons ce que nous pouvons entendre sur le sujet... ce n'est que pure facilité !

Oui, nous somme enfuis! Madame Forster et moi, oui, nous nous sommes enfuis ! ... Mais tout le monde s'est enfui ! ils couraient comme des lapins, je les vois encore, je nous vois...(... $)^{39}$ 
En outre, l'un des principaux thèmes de Lagarce est l'impossible communication des personnages. Chaque personnage dévoile son histoire dans un monologue qui est interrompu et parfois contredit par d'autres monologues car il n'existe pas un véritable échange de récits entre eux. Il n’y a plus de linéarité. La parole se divise à cause de plusieurs voix. Tous ces procédés sont les caractéristiques les plus connues du théâtre de Lagarce.

\section{Conclusion}

A l'instar de nombreux dramaturges, Lagarce n'hésite pas à s'inspirer des œuvres de différents auteurs et de faire revivre leurs personnages dans un contexte différent, ce qui lui a permis de développer son propre théâtre. S'inspirant du Journal de l'année de la peste de Defoe, Lagarce donne un tableau réel et vivant des ravages de la peste et évoque des souvenirs pénibles à partir d'une œuvre étrangère considérée comme un travail historique et documentaire.

Grâce aux traductions de cette œuvre anglaise, sorte de chronique ou de documentaire romancé sur les malheurs et les dégâts engendrés par la peste à Londres en1722, Lagarce a soigneusement décrit la souffrance physique et psychologique de ses personnages victimes de la peste qui ravageait Marseille.

Lagarce a essayé à partir de sa «relecture » du roman anglais de raconter la souffrance et la peur des personnages devant la mort. Il a utilisé la technique dramatique pour représenter les différentes préoccupations de plusieurs classes

(Notes sur d'éventuelles influences du...)Dr. Rihame Hifnawy 
sociales. A travers leurs monologues les personnages de Lagarce retracent les moments terribles et inoubliables

Pendant les jours de la peste. Ils revisitent leur passer et cherchent, en vain à effacer ce passé difficile.

Lagarce en tant que dramaturge habile a réussi à semer dans son travail l'âme du roman de Daniel Defoe en donnant aux lecteurs du XXe siècle les indices de son crime à l'intérieur du texte, notons, pour terminer, que Lagarce a dit durant son entretien avec Jean Michel Potiron que «l'auteur est un criminel, il a laissé à l'intérieur de son texte les indices de son crime ${ }^{40}$. 


\section{Les référence}

${ }^{1}$ Jacques Baillon a dit aussi qu «il est devenu à la mode et a pris un peu la place de Bernard-Marie Koltès ». Il était «l'un des auteurs les plus représentés dans l'Hexagone» Nathalie Simon, les auteurs les plus joués au théâtre in le Figaro.fr consulté le 2 juin 2018 sur internet http://www.lefigaro.fr/theatre/2008/11/27/03003-

20081127ARTFIG00484-les-auteurs-les-plus-joues-au-theatre-.php

En 2007, à l'occasion de célébrer le cinquantième anniversaire de Lagarce, quelques conférences ont été organisées dans les universités, et Les Solitaires Intempestifs, la maison d'éditions créée par Lagarce et son assistant François Berreur en 1992, a publié ces conférences. À ce moment le public se tourne vers cet auteur méconnu.

3 Jean-Pierre Thibaudat, Parcours de Jean-Luc Lagarce, consulté le 3 septembre 2018 sur internet https $/ /$ www.theatrecontemporain.net/biographies/Jean-Luc-

\section{Lagarce/ensavoirplus/idcontent/1320}

4 Daniel Defoe, de son vrai nom Daniel Foe, est un aventurier, commerçant, agent politique et écrivain anglais, né vers 16601 à Londres et mort le 24 avril 1731 dans la Cité de Londres. Il est notamment connu pour être l'auteur de Robinson Crusoé et de Heurs et Malheurs de la fameuse Moll Flanders.

5 Dans le théâtre de Samuel Becket, à titre d'exemple, à savoir que l'homme ne naît que pour mourir, ce que Beckett, qui médite dans toute son œuvre sur les fins dernières. Samuel Beckett, oh, les beaux jours, Paris, éditions de Minuit, 1963, p.45.

${ }^{6}$ L'œuvre de Lagarce se caractérise par un certain nombre de pratiques de réécriture: en 1993, Lagarce a donné Nous, les héros inspirée de Kafka et en 1980 il a écrit voyage de Madame Knipper vers la Prusse qui fait référence à Tchékhov.

7 Lagarce Jean-Luc, Journal, tome I (1977-1990), Besançon, Les solitaires Intempestifs, 2007, p.65.

8 (Henry Foe), oncle de l'auteur effectivement présent pendant l'épidémie londonienne de 1665.

${ }^{9}$ Daniel Defoe, Journal de l'année de la peste, traduction et préface de Joceph Aynard, Paris, éditions Montaigne, 1943, p.25.

10 DACHEZ Hélène, Pestes, texte et contagion : Le Journal de l'année de la peste (1722) de Daniel Defoe, in Dix-Huitième siècle

(Notes sur d'éventuelles influences du...)Dr. Rihame Hifnawy 
2015/1, N $\quad \mathrm{N}^{\circ} 47$, consulté le 4 octobre 2017 sur https://www.cairn.info/revue-dix-huitieme-siecle-2015-1-page-

311.htm?contenu=resume .

${ }_{12}^{11}$ Journal de l'année de la peste, op.cit, p. 31

12 Ibid, p.57.

${ }^{13}$ Ibid, p.56.

${ }^{14}$ Ibid, p.69.

15 LAVOCAT (François), les récits de catastrophe entre histoire et fiction (XVIe-XVIIe), in Peste, incendies, naufrages : écritures du désastre au dix-septième siècle, consulté le 4 octobre 2017 sur http://narratologie.ehess.fr/les-recits-de-catastrophes-entre-histoire-et-

fiction-xvie-xviiie-siecles-francoise-lavocat/

16 Jean-Luc Lagarce, Vagues souvenirs de l'année de la peste, in Théâtre complet II, Besançon, Les solitaires Intempestifs, 2000, p.13.

17 Ibid, p.13.

${ }^{18}$ Ibid, p. 13

${ }^{19}$ Ibid, p. 26

${ }^{20}$ Ibid, p. 19

${ }^{21}$ Ibid, p.29

${ }^{22}$ Ibid, p. 29

${ }^{23}$ Journal de l'année de la peste, op.cit, p.59

24 Le grand incendie de Londres est un violent incendie qui ravage le centre de la ville de Londres du dimanche 2 septembre au mercredi 5 septembre 1666. Les flammes ravagent la Cité à l'intérieur du mur romain et menacent le quartier aristocratique de Westminster, le palais de Whitehall, résidence du roi Charles II, et la plupart des quartiers pauvres de banlieue, sans toutefois les atteindre. Il brûle 13200 maisons, 87 églises paroissiales, la cathédrale Saint-Paul, et la majorité des bâtiments publics de la Cité. Les pertes humaines enregistrées sont très faibles, mais ne représentent peut-être qu'une fraction des victimes. Voir à cet égard Jacques Roubaud, Le Grand Incendie de Londres, Paris, Seuil, Coll. Fiction \& Cie, 1989.

${ }^{25}$ Vagues souvenirs de l'année de la peste, op.cit, p.12.

${ }^{26}$ Ibid., p.13.

${ }^{27}$ Ibid., p.38.

28 Journal de l'année de la peste, op.cit., p.36

${ }^{29}$ Daniel Defoe explique que par une corruption de mots, assez commune en Angleterre, ce nom devint Robinson Crusoé.

(Notes sur d'éventuelles influences du...)Dr. Rihame Hifnawy 
${ }^{30}$ Robinson Crusoé de Daniel Defoe a été traduit dans presque toutes les langues, y compris le copte et l'esquimau. La traduction la plus connue de Robinson Crusoé de Defoe en langue française est celle réalisée par le poète et écrivain romantique frénétique Petrus Borel au $\mathrm{XIX}^{\circ}$ siècle. Il convient de noter que Jean-Jacques Rousseau a chanté les louanges de ce roman "qui sera tout à la fois l'amusement et l'instruction d'Émile ». Rousseau a dit qu' «Il existe un livre qui fournit à mon gré le plus beau traité d'éducation naturelle, ce livre sera le premier que lira mon Emile, seul il composera durant longtemps toute sa bibliothèque et dans les temps il y tiendra une place distinguée » Voir à cet égard : Rémy Hildebrand, Héros de Jean Jacques Rousseau consulté le 2 février 2019 sur https://www.tdg.ch/reflexions/robinsoncrusoe-heros-jeanjacques-rousseau/story/19391806 , et Bernard Reymond, Robinson Crusoé, un mythe in Evangile et Liberté, Paris, $\mathrm{N}^{\circ}$. 196, février 2006 consulté le 19 mars 2018 sur www.evangile-etliberte.net/elements/numeros/196/article9.html

${ }^{31}$ Vagues souvenirs de l'année de la peste, op.cit., p.41.

32 Ibid., pp.21-22.

33 Ce roman s'inspire de la vie de Moll King (son vrai nom est Elizabeth Adkins» une criminelle de Londres que Daniel Defoe a rencontrée alors d'une visite de la prison de Newgate (prison de Londres au XVIII ${ }^{\circ}$ siècle). Ce roman a publié en 1722 mais sans doute l'idée lui en vint pendant son emprisonnement d'un an et demi à Newgate en 1704. Daniel Defoe, Heurs et Malheurs de la Fameuse Moll Flanders, traduction de Marcel Schweb, Paris, Georges Crès, 1895, p.X.

${ }^{34}$ Vagues souvenirs de l'année de la peste, op.cit., p.34.

35 Ibid., p.35.

${ }^{36}$ Ibid., p. 43-44.

${ }^{37}$ Journal de l'année de la peste, op.cit., p.59.

${ }^{38}$ M. Raimond, La crise du roman, Paris, José Corti, 1967, p. 266.

${ }^{39}$ Vagues souvenirs de l'année de la peste, op.cit., pp.18-19.

${ }^{40}$ Voir Atteindre le centre, entretien avec Jean Michel Potiron, in JeanLuc Lagarce, revue Europe, 969-970, Janvier-Février, PP.147-148. 


\section{Bibliographie}

\section{Corpus :}

1. Defoe (Daniel), Journal de l'année de la peste, traduction et préface de Joceph Aynard, Paris, éditions Montaigne, 1943.

2. Lagarce (Jean-Luc), Vagues souvenirs de l'année de la peste, in Thêâtre complet II, Besançon, Les solitaires Intempestifs, 2000.

\section{Autres œuvres des auteurs}

1. Defoe (Daniel), Heurs et Malheurs de la Fameuse Moll Flanders, traduction de Marcel Schweb, Paris, Georges Crès, 1895.

2. Lagarce (Jean-Luc), Journal, tome I (19771990), Besançon, Les solitaires Intempestifs, 2007.

Articles et entretien portant sur Jean Luc Lagarce

1. Lagarce (Jean-Luc), Atteindre le centre, entretien avec Jean Michel Potiron, in Jean-Luc Lagarce, revue Europe, 969-970, JanvierFévrier, PP.147-148.

2. Simon (Nathalie), les auteurs les plus joués au thêâtre in le Figaro.fr consulté le 2 juin 2018 sur internet http://www.lefigaro.fr/theatre/2008/11/27/03 003-20081127ARTFIG00484-les-auteurs-lesplus-joues-au-theatre-.php

3. Thibaudat (Jean-Pierre), Parcours de Jean-Luc Lagarce, consulté le 3 septembre 2018 sur internet https://www.theatrecontemporain.net/biographies/Jean-Luc-

Lagarce/ensavoirplus/idcontent/1320

\section{Articles portant sur Daniel Defoe}

1. Dachez (Hélène), Pestes, texte et contagion : Le Journal de l'année de la peste (1722) de

(Notes sur d'éventuelles influences du...)Dr. Rihame Hifnawy 
Daniel Defoe, in Dix-Huitième siècle 2015/1, $N^{\circ} 47$, consulté le 4 octobre 2017 sur https://www.cairn.info/revue-dix-huitiemesiecle-2015-1-page-311.htm?contenu=resume

2. Hildebrand (Rémy), Héros de Jean Jacques Rousseau consulté le 2 février 2019 sur https://www.tdg.ch/reflexions/robinsoncrusoe-heros-jeanjacquesrousseau/story/19391806

3. Lavocat (François), les récits de catastrophe entre histoire et fiction (XVIe-XVIIe), in Peste, incendies, naufrages : écritures du désastre au dix-septième siècle, consulté le 4 octobre 2017 sur http://narratologie.ehess.fr/les-recitsde-catastrophes-entre-histoire-et-fiction-xviexviiie-siecles-francoise-lavocat/

4. Reymond (Bernard), Robinson Crusoé, un mythe in Evangile et Liberté, Paris, $\mathrm{N}^{\circ}$. 196, février 2006 consulté le 19 mars 2018 sur www.evangile-et-

liberte.net/elements/numeros/196/article9.ht $\underline{\mathrm{ml}}$

\section{Ouvrages généraux}

1. Beckett (Samuel), oh, les beaux jours, Paris, éditions de Minuit, 1963.

2. Raimond (Michel), La crise du roman, Paris, José Corti, 1967.

3. Roubaud (Jacques), Le Grand Incendie de Londres, Paris, Seuil, Coll. Fiction \& Cie, 1989. 


\section{ملخص}

جان لوك لاجارس احد اكبر الكتاب المسرحيين المعاصرين في فرنسا ـ عرف في حياته كمخرج بارع و لم ينل قدر كبير من الثهرة ككاتب مسرحي إلا بعد وفاته. عاش اللمسرح وكتب ثماني و عشرين مسرحية. استوحى مسرحيته " ذكريات غامضة عن عام الطاعون " من رواية شهيرة للكاتب الإنجليزي دانيل ديفو و هي " يوميات عام الطاعون" و التي نشرت في السابع عشر من مارس عام IVY بتوضيح نقاط التلاقي بين المسرحية والرواية و ندرس أيضا كيف نجح لاجارس في تحويل هذا العمل الروائي إلى مسرحية ونحاول أن نعرض خصائص كل عمل مع التركيز على نقاط التشابه و الاختلاف. 